FORMATION Formation emploi

Revue française de sciences sociales

118 | avril-juin 2012

10 ans de parcours professionnels des jeunes :

l'intérêt des études longitudinales

\title{
Présentation de l'ouvrage de Christophe Guibert \& Hassen Slimani : Emplois sportifs et saisonnalités. L'économie des activités nautiques : enjeux de cohésion sociale
}

Marilyne Colombo

\section{OpenEdition \\ Journals}

Édition électronique

URL : http://journals.openedition.org/formationemploi/3658

DOI : 10.4000/formationemploi.3658

ISSN : 2107-0946

Éditeur

La Documentation française

Édition imprimée

Date de publication : 30 juin 2012

Pagination : 147-151

ISSN : 0759-6340

Référence électronique

Marilyne Colombo, « Présentation de l'ouvrage de Christophe Guibert \& Hassen Slimani : Emplois sportifs et saisonnalités. L'économie des activités nautiques : enjeux de cohésion sociale », Formation emploi [En ligne], 118 | avril-juin 2012, mis en ligne le 24 juillet 2012, consulté le 30 octobre 2020. URL http://journals.openedition.org/formationemploi/3658; DOI : https://doi.org/10.4000/

formationemploi.3658 


\title{
Note de lecture
}

\section{Présentation de l'ouvrage de Christophe GUIBERT \& Hassen SLIMANI :}

\author{
Emplois sportifs et saisonnalités. \\ L'économie des activités nautiques: \\ enjeux de cohésion sociale
}

Par Marilyne CoLombo Étudiante en Master 2 " Sport et Sciences Sociales, Administration Territoire Intégration », à l'université de Bretagne Occidentale, Brest

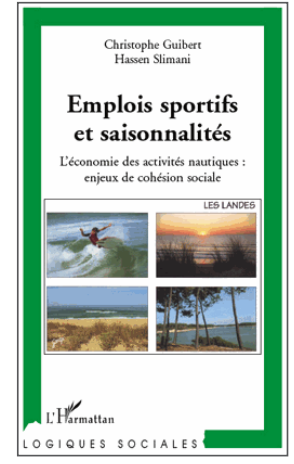

Cet ouvrage propose une analyse sociologique de la saisonnalité de l'emploi sportif propre aux activités nautiques. Il s'appuie sur un rapport commandité, en 2009, par l'Observatoire des métiers de l'animation et du sport en Aquitaine, à l'initiative du Directeur départemental de la Cohésion sociale et de la Protection des populations des Landes.

Les auteurs s'interrogent sur les mécanismes de l'emploi saisonnier dans ce secteur nautique landais. Ils observent d'une part, la manière dont les acteurs associatifs, politiques et économiques appréhendent ces emplois, et d'autre part, ils mettent en évidence les enjeux qu'ils représentent. En effet, le département des Landes se classe en quatrième position de l'emploi total des postes saisonniers dans les secteurs d'activités liés au tourisme en Aquitaine (soit 10,6 \% de l'emploi salarié derrière la Gironde, $48 \%$, les Pyrénées Atlantiques, 20,8 \% et la Dordogne, 11,1\%). Toutefois, l'ouvrage est traversé par le souci d'objectiver les effets des variations saisonnières afin d'adapter chaque pratique à l'économie touristique. À cet effet, le croisement des données statistiques locales et nationales est complété de 70 entretiens et observations in situ. Ces travaux contribuent à mieux appréhender le potentiel du secteur sportif en matière de développement économique, de création d'emplois, mais également à comprendre les difficultés relatives à une normalisation de ces emplois. 
La première partie de l'ouvrage présente les impacts structurants de l'activité touristique sur les conditions de pratique du surf, caractérisées, sur le plan économique, par une alternance entre "vide » et " plein ». Première destination d'Aquitaine, choisie par deux millions de touristes chaque année, proche de l'Espagne et riche de $106 \mathrm{~km}$ de côtes, ce département singulier des Landes a été examiné en particulier au travers de trois communes. Ainsi, maillage territorial, ressources uniques et variabilité des offres de pratique des sports nautiques ont orienté les choix vers Biscarosse, Soustons et Capbreton. Le surf représente un véritable vecteur de promotion et de développement des emplois sportifs saisonniers, mais également d'exécution de la politique territoriale (aménagement du territoire, projets sportifs territoriaux).

Un certain nombre d'indicateurs permettent de mettre en évidence la précarité des emplois sportifs, la durée des contrats, la saisonnalité, la mobilité professionnelle. Le caractère éphémère des emplois ainsi décrits, au sein de différents secteurs associatifs ou marchands, est également analysé au regard du fonctionnement des parties prenantes (associations et clubs sportifs ainsi que les différents points de vente de services et matériels spécialisés). Ces dernières proposent une offre variée de services (stages, séjours, initiation avec prêt de matériel, etc.) qui certes constitue un atout, en ajustant les offres aux demandes de la clientèle, mais également un frein au développement d'un emploi pérenne; et ce, malgré les différents dispositifs mis en place (Plan Sport Emploi, groupement national Profession Sport). En effet, par exemple, l'ouverture de nombreux points de vente de matériels et de services à proximité des lieux de pratique de surf va permettre de réaliser un chiffre d'affaires conséquent et suffisant pour ces entreprises qui ferment leurs portes dés le mois de septembre.

Les activités nautiques décrites révèlent une cristallisation spatiale des pratiques, essentiellement sur la zone du littoral, ainsi qu'une forte densité des pratiquants en période estivale. Cet univers nautique hétérogène et politiquement attractif positionne donc les Landes comme un lieu privilégié de " tourisme littoral », représentant un levier important de développement local.

Dans la deuxième partie, les auteurs s'intéressent aux activités à la périphérie de cette économie touristique, telles l'aviron, la pêche en eau douce ou en mer, le sauvetage et le secourisme, qui se heurtent au monopole territorial du surf. En appliquant aux données recueillies la même grille de lecture que précédemment, ils pointent des problématiques et des enjeux similaires de développement du tourisme landais, en portant toutefois un nouveau regard sur les institutions fédérales de tutelle. Linstauration effective de stratégies de marchandisation, en réponse à la demande d'activités estivales de " tourisme sportif ", se heurte à la problématique de renouvellement des licencié(es) au sein des fédérations mais aussi à la pérennité des pratiques compétitives. Les fédérations n’occupent plus la première place face aux besoins des clubs sportifs, confrontés à d'autres logiques de concurrence et de rentabilité. Par ailleurs, cette tension entre le fédéral et le commercial n’impacte pas les activités nautiques de manière homogène. Si ce rapport de force constitue un obstacle 
pour la voile et le canoë-kayak, en opposant et/ou en faisant se chevaucher une saison estivale et fédérale, il a permis de développer des stratégies innovantes d'actions en faveur, par exemple, du public handicapé (handi-voile) ou scolaire (canoë scolaire et surf).

La troisième partie de l'ouvrage compare trois activités, au centre de l'animation et de l'économie du tourisme landais : canoë-kayak, surf et voile. Premier paramètre essentiel, la professionnalisation des dirigeants bénévoles d'association s'impose face aux évolutions de gestion et de stratégie de développement vers des logiques entrepreneuriales. En effet, l'évolution du secteur associatif positionne les dirigeants entre bénévolat et logique d'entreprise, phénomène également étudié par certains auteurs (Loirand, 2003) ${ }^{1}$. Le fonctionnement associatif est progressivement devenu un véritable objet d'études pour les sociologues. En parallèle, de nouvelles demandes des pratiquants en matière d'activité obligent les animateurs sportifs à s'adapter. Les besoins sportifs ne sont plus liés obligatoirement à une recherche de dépassement de soi ou à l'acquisition de savoir-faire techniques. Le profil de ce nouveau pratiquant s'inscrit dans une recherche de plaisir, de sensation par une découverte immédiate de l'activité. La pratique du surf illustre bien cette transformation. L'objectif, pour le pratiquant, sera d'être capable de se tenir rapidement debout sur la planche. L'apprentissage doit être rapide et peu technique, souvent sans besoin d'acquisitions techniques supplémentaires puisque la pratique est ponctuelle. Les animateurs sportifs s'éloignent ainsi sensiblement des compétences techniques (liées à leurs pratiques) acquises en formation, pour intégrer d'autres domaines (communication, gestion, comptabilité). Ce glissement vers des missions d'agent de développement s'impose comme une nécessité pour les associations.

La comparaison des trois activités (canoë-kayak, surf et voile) pointe les enjeux de cette redéfinition d'une légitimité professionnelle qui opposent alors les brevetés d'État (plus tournés vers les activités compétitives fédérales non commerciales) et les BPJEPS (brevet professionnel de la jeunesse, de l'éducation et du sport), dédiés à l'animation d'une clientèle touristique solvable. Ainsi, le développement touristique passe par de nouvelles stratégies de "formation/emploi " qui supposent des compléments de formation visant à occuper les temps "vide " du métier de moniteur. Comment alors répondre à des besoins variés, généraliser une réponse au regard des profils sociologiques multiples des moniteurs sportifs, d'une activité nautique à l'autre ? La question de la "précarité " des moniteurs doit être envisagée sous un autre angle. En effet, la fragilité professionnelle, la commercialisation et la "desportivisation » des pratiques (pratiques de loisirs qui nécessitent peu d'apprentissage et d'encadrement technique, accessibles rapidement), le décalage entre formation et fiche de poste sont autant de constats qui ne suscitent pas pour autant de demande coordonnée et récurrente de la part des principaux intéressés. Le monde associatif sportif incarne des valeurs qui ne se veulent pas dominées par le souci du profit individuel et de l'enrichissement personnel, mais par celui de l'intérêt général, de la citoyenneté, du

1. Les paradoxes de la "professionnalisation" des associations sportives, Rennes, Presses universitaires, 2003. 
partage des richesses, de la cohésion sociale $\left(\right.$ Chanial, 1998) ${ }^{2}$. Les exemples choisis par les auteurs ne témoignent pas d'une demande généralisable d'accès au plein emploi.

Cette analyse des mécanismes de la saisonnalité des emplois permet donc de dépasser le simple constat d'instabilité voire de "précarité " qui sert de lieu commun. Elle offre une vision plus précise en prenant en compte divers indicateurs et points de vue (élus, dirigeants du secteur associatif sportif et touristique). On notera la pertinence de l'analyse des enjeux de politiques territoriales - commerciaux, éducatifs et sociaux - réalisée ici au travers des activités nautiques landaises. De ce fait, les transpositions à d'autres disciplines ou territoires deviennent possibles. En effet, un territoire de montagne sur lequel se pratiquent des sports alpins très divers pourrait faire l'objet d'une étude similaire.

Au-delà des principaux constats, les préconisations formulées par les auteurs visent à combiner les effets des variations saisonnières de et sur l'emploi sportif. L'appropriation des savoirs et savoir-faire extra-sportifs serait la condition sine qua non permettant aux animateurs sportifs de tendre vers le plein emploi.

Par ailleurs, si les différentes évolutions observées sont explicitées par les auteurs, l'implication des institutions reste à explorer, en particulier sur les stratégies de développement territorial en cohérence avec l'évolution de l'emploi sportif. Les conclusions n'éclairent pas sur les choix politiques, économiques et sportifs des institutions en charge de l'animation territoriale de loisirs hors saison. La mise en œuvre de la " cohésion sociale ", définie par Emile Durkheim comme un état de bon fonctionnement de la société, renvoie à l'importance des enjeux économiques et touristiques pour ces collectivités chargées d'assurer le bien-être de tous leurs membres, en réduisant les disparités et en évitant la marginalisation. Lobservation des activités nautiques sur ce territoire positionne bien ce secteur comme un levier majeur de développement économique. Ainsi, l'emploi sportif est devenu un champ en développement de recherches en sciences sociales (Falcoz et Walter, 2009) ${ }^{3}$, riche en enseignements.

L'espace territorial ainsi partagé entre collectivités, associations, clubs et secteur marchand, nécessite une réflexion commune qui permettra à chaque acteur d'y trouver un ancrage profitable, économique, politique, socio-éducatif. L'exemple du surf illustre l'évolution d'un sport pluriel, tantôt produit d'appel pour la notoriété d'une collectivité, tantôt outil de cohésion sociale pour les institutions de l'État, tantôt encore produit socio-éducatif dans le cadre des partenariats scolaires. Le développement économique et touristique autour d'une activité sportive doit s'envisager dans un ensemble varié. Ainsi, les acteurs des secteurs marchand, collectif ou associatif peuvent se l'approprier.

2. La délicate essence de la démocratie, MAUSS, $\mathrm{n}^{\circ}$ 11, 1998.

3. "Être salarié dans un club sportif : une posture problématique ", Formation Emploi 4/2009, n 108, pp. 25-37. 
Toutefois, l'analyse est examinée au regard de la commande (pour rappel, commande de l'Observatoire des métiers de l'animation et du sport en Aquitaine). Selon les auteurs, la Révision Générale des Politiques Publiques ${ }^{4}$ explique, en grande partie, l'intérêt croissant des élus locaux et des services de l'État commanditaires à l'égard de ces activités nautiques sur le territoire des Landes. Le repositionnement des instances locales à l'aune de cette réforme a donc suscité un regain d'intérêt pour ces emplois sportifs. La réorganisation des services de l'État, et en particulier la disparition de l'échelon départemental du ministère des Sports et de la Santé, a conduit à éloigner les responsables des réalités du terrain, dont l'analyse parait pourtant indispensable à l'action.

Cependant, si les enjeux politiques et sociaux de la saisonnalité sont classiques - mise en œuvre de politiques sportives territoriales, préservation de l'emploi et des formations - les auteurs illustrent et renforcent l'idée que les activités sportives ont toujours été vectrices de socialisation et de régulation sociale. Les pratiques sportives ont en effet toujours représenté des lieux et occasions de mixité sociale, par l'intégration de notions de dépassement de soi, de solidarité ou d'assimilation des différences.

L'intérêt de cette enquête réside ainsi dans une interrogation : la saisonnalité de ces emplois met-elle en péril la cohésion sociale ? Au-delà d'un diagnostic territorial, les préconisations propres à développer l'emploi sportif et renforcer la cohésion sociale y trouvent une légitimité institutionnelle dans les objectifs d'une réforme territoriale.

Cet ouvrage reflète le sens des débats actuels dans les territoires, où la variété des représentations des activités nautiques, des statuts juridiques des organisations, des profils sociologiques des moniteurs sportifs oblige à traiter l'emploi sportif comme un facteur de cohésion sociale parmi d'autres, structurant pour un territoire. La relation formationemploi permettrait de répondre aux demandes variées sur le terrain et de combler les besoins des structures. Ainsi, la professionnalisation montante dans le sport fédéral et associatif ouvre des perspectives et fait déjà l'objet de nombreuses études (Le Roux, 2002)5. Outre le caractère durable ou non de l'employabilité dans ce secteur, le repérage de secteurs et d'emplois émergents sur les territoires constitue en définitive un enjeu central.

\section{Référence de l'ouvrage}

Guibert Christophe \& Slimani Hassen (2011), Emplois sportifs et saisonnalités. L'économie des activités nautiques : enjeux de cohésion sociale, Paris, L'Harmattan, 242 p.

4. La Révision Générale des Politiques Publiques (RGPP), programme de modernisation de l'action de l'État touchant l'ensemble des politiques publiques et l'ensemble des ministères, lancée en juin 2007.

5. L'emploi sportif en France : situation et tendances d'évolution, AFRAPS/RUNOPES. 